\title{
Congenital thiopurine methyltransferase deficiency and 6-mercaptopurine toxicity during treatment for acute lymphoblastic leukaemia
}

\author{
L Lennard, B E S Gibson, T Nicole, J S Lilleyman
}

\begin{abstract}
Two children with acute lymphoblastic leukaemia (ALL) taking daily 6-mercaptopurine as part of a national UK therapeutic trial repeatedly developed profound myelosuppression on $25 \%$ of the standard protocol dose. Both were found to have undetectable intracellular activity of thiopurine methyltransferase (TPMT), an enzyme controlling one of the major alternative catabolic pathways of 6-mercaptopurine, and both produced higher concentrations of cytotoxic drug metabolites at $10-25 \%$ of the protocol dose than other patients taking $100 \%$.

It is supposed that these patients represent the $0.33 \%$ of the normal population constitutionally lacking TPMT. It is important to recognise such individuals both to avoid fatal bone marrow failure through inadvertent overdosage, and to be reassured that an adequate drug effect can be achieved at around $10 \%$ of the standard dose.
\end{abstract}

(Arch Dis Child 1993; 69: 577-579)

Inherited as a genetic trait, constitutional absence of thiopurine methyltransferase (TPMT) activity occurs in $0.33 \%$ of the white population ${ }^{1}$ and so could be expected to occur in a similar proportion of children being treated with 6-mercaptopurine for acute lymphoblastic leukaemia (ALL).

The enzyme is responsible for $\mathrm{S}$-methylation of 6-mercaptopurine and its initial nucleotide metabolites, and competes with two other 6-mercaptopurine metabolic pathways, modulated by hypoxanthine phosphoribosyl transferase (HPRT) and xanthine oxidase respectively.

6-Mercaptopurine is a prodrug with no intrinsic cytotoxic activity. It has to be processed within cells to produce cytotoxic metabolites, the most important being the 6thioguanine nucleotides at the end of the HPRT pathway. Intracellular concentrations of these metabolites correlate with granulocytopenia during 6-mercaptopurine treatment, ${ }^{23}$ and low concentrations seen during treatment of ALL appear to be associated with a higher risk of relapse. ${ }^{4}$

6-Mercaptopurine is broken down by xanthine oxidase, and it has long been known that the concurrent administration of allopurinol (a xanthine oxidase inhibitor) with 6-mercaptopurine potentiates cytotoxicity. ${ }^{56}$ Less well recognised, but no less clinically important, low or absent TPMT activity is also associated with grossly raised 6-thioguanine nucleotide concentrations and profound myelosuppression after only a brief exposure to thiopurine drugs. ${ }^{7}$ The problem has been seen in occasional adults on immunosuppressive treatment with azathioprine (a 6-mercaptopurine prodrug), ${ }^{78}$ and has been described in children with ALL. 910

We have recently encountered two children in the UK ALL trial series who gave an opportunity to assess intracellular drug metabolites in such circumstances and to explore how best the rare child with ALL and constitutional TPMT deficiency might be managed. The values were compared with a well studied unselected cohort of children with intermediate or high TPMT activity.

\section{Methods and reference ranges \\ REFERENCE POPULATION}

Ranges for 6-methylmercaptopurine metabolites (MeMPs), 6-thioguanine nucleotides, and TPMT activity were taken from a consecutive cohort of 25 children with ALL from two treatment centres. Assays were taken during continuation chemotherapy on current UK ALL protocols where prolonged treatment with daily 6-mercaptopurine is coupled with weekly methotrexate and monthly vincristine plus steroids. Samples were taken at routine venepuncture at a time when the patients had been prescribed $100 \%$ standard protocol dose of 6-mercaptopurine $\left(75 \mathrm{mg} / \mathrm{m}^{2}\right)$ for at least four weeks or more and when no red cell transfusions had been given for at least three months.

ASSAYS

Red blood cell concentrations of MeMPs, products of the TPMT reaction, and 6thioguanine nucleotides were measured as previously described. ${ }^{11}$ Red blood cell TPMT activity was measured by a slight modification of the method of Weinshilboum et al. ${ }^{11} 12$ One unit of TPMT activity represents the formation of 1 nmol 6-methylmercaptopurine per hour of incubation per ml of packed red blood cells.

REFERENCE VALUES

6-Thioguanine nucleotide concentrations ranged from $144-1263 \mathrm{pmol} / 8 \times 10^{8} \mathrm{red}$ blood cells (median 335), MeMP concentrations ranged from $0 \cdot 25-22.5 \mathrm{nmol} / 8 \times 10^{8}$ red blood 
6-Mercaptopurine dosages, 6-thioguanine nucleotide concentrations (pmol/8 $\times 10^{8}$ red blood cells) MeMP concentrations ( $\mathrm{nmol} / 8 \times 10^{8}$ red blood cells), and TPMT activities (units $/ \mathrm{ml}$ red blood cells) in the reference population (control group) compared with patients 1 and 2 $\left(100 \%\right.$ 6-mercaptopurine $\left.=75 \mathrm{mg} / \mathrm{m}^{2}\right)$.

\begin{tabular}{|c|c|c|c|}
\hline \% 6-Mercaptopurine & $\begin{array}{l}\text { Range (median) } \\
\text { 6-thioguanine } \\
\text { nucleotides }\end{array}$ & $\begin{array}{l}\text { Range (median) } \\
\text { MeMPs }\end{array}$ & $\begin{array}{l}\text { Range (median) } \\
T P M T\end{array}$ \\
\hline \multicolumn{4}{|l|}{ Control group } \\
\hline $100 \% \times 4$ weeks & $144-1263(335)$ & $0.25-22.5(4.36)$ & $9 \cdot 2-25 \cdot 1(17 \cdot 5)$ \\
\hline \multicolumn{4}{|l|}{ Patient 1} \\
\hline $25 \% \times 2$ weeks & 1832 & Nil & \\
\hline $10 \% \times 3$ weeks & 960 & Nil & \\
\hline $10 \% \times 6$ weeks & 1701 & Nil & $<0.74$ \\
\hline \multicolumn{4}{|l|}{ Patient 2} \\
\hline $50 \% \times 1$ week & 1601 & Nil & \\
\hline $0 \% \times 1$ week & 2650 & Nil & \\
\hline $25 \% \times 2$ weeks & 2250 & Nil & \\
\hline $0 \% \times 3$ weeks & 628 & Nil & $<0.074$ \\
\hline
\end{tabular}

cells (median $4 \cdot 36$ ), and TPMT values ranged from $9 \cdot 2-25 \cdot 1$ units (median $17 \cdot 5$ ).

\section{Case reports}

PATIENT 1

A 3 year old boy developed 'common' ALL and was treated according to the then current UK protocol, UKALL XA. He entered remission uneventfully, and started 6-mercaptopurine treatment on schedule at $100 \%$ dose (75 $\mathrm{mg} / \mathrm{m}^{2}$ ). After 19 days he developed neutropenia (neutrophil count $0.2 \times 10^{9} / 1$ ) and thrombocytopenia (platelet count $12 \times 10^{9} / 1$ ) became unwell and needed admission to hospital. His counts recovered during the ensuing six weeks, and 6-mercaptopurine was restarted at $50 \%$ dose rising to $75 \%$ over four weeks. At this stage his counts plummeted again (neutrophils $0.5 \times 10^{9} / 1$, platelets $\left.12 \times 10^{9} / 1\right)$. Subsequently he never tolerated more than $25 \%$ of the protocol dose of 6mercaptopurine.

6-Mercaptopurine metabolites were measured on three occasions: after two weeks at $25 \%$ protocol dose (6-thioguanine nucleotides $1832 \mathrm{pmol} / 8 \times 10^{8}$ red blood cells, MeMPs nil), after three weeks at $10 \%$ protocol dose (6-thioguanine nucleotides $960 \mathrm{pmol} / 8 \times 10^{8}$ red blood cells, MeMPs nil), and after six weeks at $10 \%$ protocol dose (6-thioguanine nucleotides $1701 \mathrm{pmol} / 8 \times 10^{8}$ red blood cells, MeMPs nil). TPMT activity was recorded at $<0.74$ units, the lowest detectable activity in the assay used (table).

He settled to a tolerated $10 \%$ of protocol dose, though his platelet count was $<100 \times 10^{9} / 1$ for two months on one occasion, and treatment had to be interrupted four more times when his platelets fell to $<50 \times 10^{9} / 1$ and/or his neutrophils below $0.5 \times 10^{9} / 1$. He completed his treatment and continues in his first remission three years from diagnosis.

PATIENT 2

A 12 year old girl developed 'common' ALL with an initial white cell count of $196 \times 10^{9} / 1$ and was treated according to the protocol of UKALL XI. At the start of 6-mercaptopurine treatment she was prescribed full protocol dose and four weeks later was profoundly neutropenic (neutrophil count zero) and thrombocytopenic (platelet count $129 \times 10^{9} / 1$ ). Her counts recovered in a month and she was reintroduced to 6-mercaptopurine at a starting dose of $25 \%$ rising to $50 \%$ over three weeks. Pancytopenia recurred. Subsequently she never tolerated 6-mercaptopurine for more than three weeks and rarely received more than $25 \%$ of the standard dose.

6-Mercaptopurine metabolites were measured on four occasions: after 1 week at $50 \%$ of the standard dose (6-thioguanine nucleotides $1601 \mathrm{pmol} / 8 \times 10^{8}$ red blood cells, MeMPs nil), after one week at $25 \%$ (6-thioguanine nucleotides $2250 \mathrm{pmol} / 8 \times 10^{8}$ red blood cells, MeMPs nil), and also one and three weeks after withdrawing treatment at $25 \%$ (6-thioguanine nucleotides 2650 and 628 $\mathrm{pmol} / 8 \times 10^{8}$ red blood cells, MeMPs nil and nil, respectively). TPMT was assayed at $<0 \cdot 74$ units, below the limit of assay (table).

The patient settled to a cycle of daily 6mercaptopurine for three weeks at $25 \%$ of the standard dose usually followed by a cytopenia induced 3-4 week gap. She continues in her first remission 1.5 years from diagnosis.

\section{Discussion}

The two children described illustrate the problems of thiopurine drug treatment in patients constitutionally lacking TPMT. Both developed severe myelotoxicity on standard doses of 6-mercaptopurine, and both were subsequently able to tolerate only very small doses of the drug. Despite this, both consistently showed high concentrations of intracellular 6-thioguanine nucleotides, higher than those seen in normal children taking full doses.

The assumption that they lacked TPMT was based on direct assay of the enzyme and the repeated failure to detect any MeMPs - the product of the enzyme. Although MeMPs fluctuate widely in normals on 6-mercaptopurine, they are never undetectable.

TPMT deficiency occurs in approximately $0.33 \%$ of the UK population, and some 400 children each year develop ALL. Current treatment schedules run for two years, so at any one time around 800 patients will be on continuous 6-mercaptopurine. Statistically two or three will be TPMT deficient, so the two patients in this report probably represent a nationwide experience. Because of their rarity, it is possible that paediatric oncologists may not encounter a TPMT deficient child for many years, but it is important that they are aware of the possibility to avoid potentially life threatening marrow toxicity when giving 6mercaptopurine. The same would apply to children receiving azathioprine for any reason.

The concentrations of 6-thioguanine nucleotides seen in the two patients are reassuringly high, despite the fact that they were taking what might be regarded as homoeopathic doses of 6-mercaptopurine. It is likely that these cytotoxic metabolites, as chief mediators of the drug's antineoplastic effect, can be maintained at satisfactory concentrations on tiny doses in such children. They accumulate slowly in cells over several days and then disappear at the same rate, as shown 
by patient 2 , who was studied at one and two weeks after interrupting treatment. TPMT deficient children are never likely to reach 'steady state' for 6-thioguanine nucleotides, but their concentration can probably be kept within reasonable limits by judicious titration against the neutrophil and platelet count.

The fact that TPMT deficient children do not produce intracellular MeMPs may be unimportant as far as the cytotoxic effect of 6mercaptopurine is concerned, though the precise role and kinetics of those metabolites is still not clear. There appears to be an inverse correlation between 6-thioguanine nucleotides and MeMPs, ${ }^{13}$ but they may interact in cytotoxicity, as children without MeMPs appear to tolerate much higher concentrations of 6thioguanine nucleotides. This is apparent when 6-thioguanine, a direct source of the nucleotides, is offered as an alternative to 6mercaptopurine. ${ }^{14}$

The most important point about TPMT deficiency is to avoid toxicity with 6-mercaptopurine and azathioprine. But it is also important to appreciate that an apparently adequate drug effect can be achieved on markedly reduced doses.

This work was supported by the Leukaemia Research Fund of Great Britain and the Yorkshire Cancer Research Campaign.

1 Weinshilboum RM, Sladek SL. Mercaptopurine pharmacogenetics: monogenic inheritance of erythrocyte thiopurine methyltransferase activity. Am $\mathcal{f}$ Hum Genet 1980; 32: 651-62.
2 Lennard L, Lilleyman JS, Van Loon JA, Weinshilboum RM. Genetic variation in response to 6 -mercaptopurine for childhood acute lymphoblastic leukaemia. Lancet 1990; 336: 225-9.

3 Herber S, Lennard L, Lilleyman JS, Maddocks JL. 6Mercaptopurine: apparent lack of relation between prescribed dose and biological effect in children with leukaemia. $B r$ f Cancer 1982; 46: 138-41.

4 Lennard L, Lilleyman JS. Variable 6-mercaptopurine metabolism and treatment outcome in childhood lymphoblastic leukaemia. $\mathcal{f}$ Clin Oncol 1989; 7: 1816-23.

5 Brooks RJ, Dorr RT, Durie BGM. Interaction of allopurinol with 6-mercaptopurine and azathioprine. Biomedicine 1982; 36: 217-22.

6 Murrell GAC, Rapeport WG. Clinical pharmacogenetics of allopurinol. Clin Pharmacokinet 1986; 11: 343-53.

7 Lennard L, Van Loon JA, Weinshilboum RM. Pharmacogenetics of acute azathioprine toxicity: relationship to thiopurine methyltransferase genetic polymorphism. Clin Pharmacol Ther 1989; 46: 149-54.

8 Anstey A, Lennard L, Mayou SC, Kirby JD. Pancytopenia related to azathioprine - an enzyme deficiency caused by a common genetic polymorphism: a review. $\mathcal{F} R$ Soc Med 1982; 85: 752-6.

9 Evans WE, Horner M, Chu YQ, Kalwinsky D, Roberts WM. Altered mercaptopurine metabolism, toxic effects, and dosage requirement in a thiopurine methyltransferase deficient child with acute lymphoblastic leukaemia. $f$ Pediatr 1991; 119: 985-9.

10 McLeod HL, Miller DR, Evans WE. Azathioprine-induced myelosuppression in thiopurine methyltransferase deficient heart transplant recipient. Lancet 1993; 341: 1151

11 Lennard L, Singleton H. High performance liquid chromatographic assay of the methyl and nucleotide metabolites of 6-mercaptopurine: quantitation of red blood cell 6-thioguanine nucleotide, 6-thioinosinic acid and 6-methylmercaptopurine metabolites in a single sample. F Chromatog 1992; 583: 83-90.

12 Weinshilboum RM, Raymond FA, Pazmino PA. Human erythrocyte thiopurine methyltransferase: radiochemical microassay and biochemical properties. Clin Chim Acta 1978; 85: 323-33.

13 Lennard L, Lilleyman JS. Compliance with 6-mercaptopurine therapy in UKALL trials. Br $\mathcal{F}$ Haematol 1993; 84 (suppl 1): 19 .

14 Lennard L, Davies HA, Lilleyman JS. Is 6-thioguanine more appropriate than 6-mercaptopurine for children with acute lymphoblastic leukaemia? $\mathrm{Br} \mathcal{f}$ Cancer 1993 68: $186-90$. 